\title{
Appraisal on Ayurvedic Herbs in the Management of Sthaulya (Obesity)
}

\author{
Review Article
}

\section{Murali Krishna $C^{1 *}$, Sivaram $G^{2}$, Panchajanya Kumar $D^{2}$, Malini $S^{3}$, Anumol K ${ }^{1}$, Sujata P. Dhoke ${ }^{1}$, Venkateshwarlu B ${ }^{1}$, Babu $^{4}$}

\begin{abstract}
1. Research Officer (Ay), 2. Senior Research Fellow (Ay), 3. Research Officer (Bio-chemistry), 4. Assistant Director \& Incharge, RARISD, CCRAS, Vijayawada-15 India.
\end{abstract}

\begin{abstract}
Sthaulya (Obesity) is a lifestyle and metabolic disorder. Ayurveda treated Sthaulya as a Santarpanottha Vikara (diseases due to excessive nutrition) which has been identified as one of the leading cause of morbidity and mortality worldwide in both developed and developing countries. Medodushti (disorders of fat metabolism) serves as one of the important etiological factor. In Ayurveda herbal drugs are being used to lower the levels of serum cholesterol for the treatment and prevention of this disorder. In this regard, an attempt has been made to review the drugs useful in Sthaulya specifically mentioned in Ganas (group of drugs) of Ayurvedic classical texts which are useful in prevention and management of conditions like Dyslipidemia and its complications.
\end{abstract}

Keywords: Dyslipidemia, Lekhana, Medohara, Sthaulya, Obesity, Ayurveda.

\section{Introduction:}

Sthaulya (obesity) is one of the lifestyle and metabolic disorder which was explained in Ayurvedic classics. In Ayurveda it is diagnosed generally basing on the characteristic features like chala (sagging) sphik (buttocks), udara (belly) and sthana (breast), also decreased upachaya (general strength) and utsaaha (enthusiasm).

In modern the accumulation of fat over the limit led to ill/adverse effect in the body known as obesity. Body mass index (BMI) is an index of weight-forheight that is commonly used to classify overweight and obesity in adults. The World Health Organization (WHO) definition is "A BMI greater than or equal to 25 is overweight and a BMI greater than or equal to 30 is obesity".(1)

Overweight and obesity are the fifth leading risk for global deaths. At least, 2.8 million adults die each year as a result of being overweight or obese. In addition, $44 \%$ of the diabetes burden, $23 \%$ of the ischemic heart disease burden and between $7 \%$ and $41 \%$ of certain cancer burdens are attributable to overweight and obesity. WHO global estimates for the year 2008 , reported 1.5 billion people were overweight, of these, over 200 million men and nearly 300 million women were obese. Overall more than one in ten of the world's adult population was obese.(1)

In 2010, around 43 million children under five were overweight. Overweight and obesity are now on

*Corresponding Author:

Murali Krishna C

Research Officer (Ayurveda)

RARISD, CCRAS, Vijayawada-15,

E-mail: mkchagamreddy@gmail.com the rise in low and middle income countries, particularly in urban settings. Close to 35 million overweight children are living in developing countries and 8 million in developed countries. Childhood obesity is associated with a higher chance of premature death and disability in adulthood. But in addition to increased future risks, obese children experience breathing difficulties, increased risk of fractures, hypertension, cardiovascular diseases and psychological effects. (2)

Atisthaulya (over obese) is considered as one of the eight despicable conditions as described by Acharya Charaka.(3) A person in whom there is excessive accumulation of Meda (fat/adipose tissue) and Mamsa (flesh/muscle tissue) leading to flabbiness of hips, abdomen, and breast has been categorized as Atisthaulya.(4) Medas is body tissue predominant in Prithvi (earth) and Ap (water) Mahabhutas (basic elements) similar to Kapha Dosha.(5) It is characterized by Snighdha (unctuous), Guru (heavy), Sthula (space occupying), Picchila (slimy), Mridu (tender/ soft) and Sandra (dense) Guna (qualities).(6) Sneha (oleation), Sweda (production of sweat), Drudhatva (compactness), and Asthipushti (nourishment of bones) are the main function of Medodhatu.(7) Consumption of Guru (heavy to digest), Sheeta (cold), Snigdha (unctuous), Madhuradi Kaphavardhaka (sweet and Kapha increasing) drugs along with lack of exercise and sedentary life style result in excessive nourishment of Medas while other bodily elements (Dhatus) are deprived of nourishment.

Disproportionately increased Medas is accountable for several serious consequences reported in Charaka Samhita like Ayuhrasa (decrease of life span), Javoparodha (decrease in enthusiasm and activity), Krichravyavayata (difficulty in sexual act), Dourbalya (decrease of strength), Dourgandhya (bad 
odor), Swedabadha (excess perspiration) and Kshut Pipasadhikya (excessive hunger and thirst).(8) Mandotsaham (less activity referring to sedentary lifestyle), Atisnigdham (excessive intake of fatty substances), Atisthaulyam (gross obesity), and Mahashanam (excessive eating) constitute for causation of Prameha(9) (urinary diseases including Diabetes) and these etiological factors may also initiate Dyslipidemia.

Obesity and Hyperlipidemia being the most common problems in older age groups as well as adolescents, there is a necessity to combat them with drugs mentioned in Ayurvedic classics which may be useful in the conditions of Medodushti and Sthaulya. In this regard, an attempt has been made to critically review the Medohara drugs mentioned in the classical texts which may give us a better understanding for its prevention and management.

\section{Materials and Methods:}

Compilation of Medohara (which removes or dries up excess Medas) and Lekhaneeya (which scrapes excess Medas) herbs were done from Ganas of Charaka Samhita(10), Sushruta Samhita(11), Astanga sangraha
(12), and Ashtanga Hrudaya(13). A total of 100 different herbs have been enlisted from these various groups (Ganas) of classical Ayurvedic texts after excluding the controversial drugs. Rasa (taste), Guna (quality), Veerya (potency), Vipaka (drug metabolism), Action on Doshas and useful part of the herbs were compiled from Dhanvantari Nighantu,(14) Bhavaprakasha Nighantu,(15) Nighantu Adarsha,(16) and Textbook of Dravyaguna $(17,18)$ was tabulated. Herbs with proven lipid lowering activity reported through different research models are also tabulated. $(19,20,21)$

\section{Results:}

Charaka has given single Gana of 10 drugs (Lekhaniya Gana), while Sushruta and Vagbhata have mentioned 8 and 10 Ganas respectively in Table-1. Analysis of 100 drugs has been made, which are taken from different Ganas of classical texts. Useful part was recorded based on relevant references from classical texts and modern texts of Dravyaguna in Table-2. Herbs with proven lipid lowering activity are mentioned in Table- 3 .

Table 1: List of Ganas mentioned in Ayurvedic classical for Sthaulya

\begin{tabular}{|l|c|c|c|c|}
\hline Name of the Gana & C.S & S.S & A.S & A.H \\
\hline Arkadi gana & $\mathrm{x}$ & $\checkmark$ & $\checkmark$ & $\checkmark$ \\
\hline Asanadi gana & $\mathrm{x}$ & $\mathrm{x}$ & $\checkmark$ & $\checkmark$ \\
\hline Lekhaniya gana & $\checkmark$ & $\mathrm{x}$ & $\mathrm{x}$ & $\mathrm{x}$ \\
\hline Lodhradi gana & $\mathrm{x}$ & $\checkmark$ & $\checkmark$ & $\checkmark$ \\
\hline Mushkakadi gana & $\mathrm{x}$ & $\checkmark$ & $\checkmark$ & $\checkmark$ \\
\hline Nyagrodhadi gana & $\mathrm{x}$ & $\checkmark$ & $\checkmark$ & $\checkmark$ \\
\hline Salasardi gana & $\mathrm{x}$ & $\checkmark$ & $\mathrm{x}$ & $\mathrm{x}$ \\
\hline Surasadi gana & $\mathrm{x}$ & $\mathrm{x}$ & $\checkmark$ & $\checkmark$ \\
\hline Tryushana gana & $\mathrm{x}$ & $\checkmark$ & $\mathrm{x}$ & $\mathrm{x}$ \\
\hline Ushakadi gana & $\mathrm{x}$ & $\checkmark$ & $\checkmark$ & $\checkmark$ \\
\hline Vacha Haridradi gana & $\mathrm{x}$ & $\mathrm{x}$ & $\checkmark$ & $\checkmark$ \\
\hline Varunadi gana & $\mathrm{x}$ & $\checkmark$ & $\checkmark$ & $\checkmark$ \\
\hline Vatsakadi gana & $\mathrm{x}$ & $\mathrm{x}$ & $\checkmark$ & $\checkmark$ \\
\hline
\end{tabular}

Note: C.S: Charaka Samhita; S.S: Susruta Samhita; A.S: Ashtanga Sangraha; A.H: Ashtanga Hrudaya

Table 2: List of Sthaulya hara (Anti-Obesity) drugs

\begin{tabular}{|c|c|c|c|c|c|c|c|c|}
\hline $\begin{array}{c}\text { S. } \\
\mathbf{N} \\
\mathbf{0}\end{array}$ & $\begin{array}{c}\text { Sanskrit } \\
\text { name }\end{array}$ & Latin name & $\begin{array}{c}\text { Rasa } \\
\text { (taste) }\end{array}$ & $\begin{array}{c}\text { Guna } \\
\text { (qualities) }\end{array}$ & $\begin{array}{c}\text { Virya } \\
\text { (potency) }\end{array}$ & $\begin{array}{c}\text { Vipaka } \\
\text { (after } \\
\text { digestio } \\
\text { n) }\end{array}$ & $\begin{array}{c}\text { Dosha } \\
\text { passific } \\
\text { aion }\end{array}$ & $\begin{array}{c}\text { Usefu } \\
\text { I part }\end{array}$ \\
\hline 1 & Agnimantha & Premna integrifolia Linn. & $\begin{array}{c}\text { Katu } \\
\text { (pungent), } \\
\text { Tikta } \\
\text { (bitter) }\end{array}$ & $\begin{array}{c}\text { Laghu } \\
\text { (lightness), } \\
\text { Rooksha } \\
\text { (dry) }\end{array}$ & $\begin{array}{c}\text { Ushna } \\
\text { (Hot) }\end{array}$ & Katu & $\begin{array}{c}\text { Kapha, } \\
\text { Vata }\end{array}$ & $\begin{array}{c}\text { Root } \\
\text { bark, } \\
\text { leaf }\end{array}$ \\
\hline 2 & Aguru & Aquilaria agallocha Roxb. & Katu, Tikta & $\begin{array}{c}\text { Laghu, } \\
\text { Teekshna } \\
\text { (piercing) }\end{array}$ & Ushna & Katu & $\begin{array}{c}\text { Kapha, } \\
\text { Vata }\end{array}$ & $\begin{array}{c}\text { resino } \\
\text { us } \\
\text { wood, } \\
\text { oil }\end{array}$ \\
\hline 3 & Ajakarna & $\begin{array}{r}\text { Dipterocarpus turbinatus } \\
\text { Gaertn.f. }\end{array}$ & Katu, Tikta & $\begin{array}{c}\text { Laghu, } \\
\text { Snigdha } \\
\text { unctuous) }\end{array}$ & Ushna & Katu & $\begin{array}{c}\text { Kapha, } \\
\text { Vata }\end{array}$ & $\begin{array}{c}\text { Oil, } \\
\text { bark, } \\
\text { fruit }\end{array}$ \\
\hline
\end{tabular}


Murali Krishna C et.al., Appraisal on Ayurvedic Herbs in the Management of Sthaulya (Obesity)

\begin{tabular}{|c|c|c|c|c|c|c|c|c|}
\hline 4 & Ajamoda & $\begin{array}{c}\text { Trachyspermum roxburghianum } \\
\text { (DC.) Craib. }\end{array}$ & $\begin{array}{l}\text { Katu, } \\
\text { Tikta } \\
\end{array}$ & $\begin{array}{c}\text { Laghu, } \\
\text { Rooksha }\end{array}$ & Ushna & Katu & $\begin{array}{c}\text { Kapha, } \\
\text { Vata }\end{array}$ & Fruits \\
\hline 5 & Alarka & $\begin{array}{c}\text { Calotropis gigantea (Linn.) R.Br. } \\
\text { ex.Ait. }\end{array}$ & $\begin{array}{l}\text { Katu, } \\
\text { Tikta }\end{array}$ & $\begin{array}{l}\text { Laghu, } \\
\text { Rooksha }\end{array}$ & Ushna & Katu & $\begin{array}{c}\text { Kapha, } \\
\text { Vata }\end{array}$ & $\begin{array}{c}\text { Root } \\
\text { bark, } \\
\text { flowers, } \\
\text { leaf, } \\
\text { latex, } \\
\text { seeds } \\
\end{array}$ \\
\hline 6 & Amalaki & Emblica officinalis Gaertn. & $\begin{array}{c}\text { Five } \\
\text { tastes } \\
\text { (except } \\
\text { lavana) }\end{array}$ & $\begin{array}{l}\text { Guru } \\
\text { (heavines } \\
\text { s), Sheeta } \\
\text { (coolant) }\end{array}$ & Sheeta & $\begin{array}{c}\text { Madhu } \\
\text { ra } \\
\text { (sweet) }\end{array}$ & Tridosha & Fruits \\
\hline 7 & Amra & Mangifera indica Linn. & $\begin{array}{c}\text { Kashay } \\
a \\
\text { (astring } \\
\text { ent) }\end{array}$ & Laghu & Sheeta & Katu & $\begin{array}{l}\text { Kapha, } \\
\text { pitta }\end{array}$ & $\begin{array}{l}\text { Bark, } \\
\text { Seeds }\end{array}$ \\
\hline 8 & Arjuna & $\begin{array}{c}\text { Terminalia arjuna (Roxb.) W. \& } \\
A .\end{array}$ & $\begin{array}{c}\text { Kashay } \\
a\end{array}$ & $\begin{array}{l}\text { Laghu, } \\
\text { Rooksha }\end{array}$ & Sheeta & Katu & $\begin{array}{l}\text { Kapha, } \\
\text { pitta }\end{array}$ & Bark \\
\hline 9 & Arka & Calotropis procera (Ait.) R.Br. & $\begin{array}{l}\text { Katu, } \\
\text { Tikta }\end{array}$ & $\begin{array}{l}\text { Laghu, } \\
\text { Rooksha }\end{array}$ & Ushna & Katu & $\begin{array}{c}\text { Kapha, } \\
\text { Vata }\end{array}$ & $\begin{array}{c}\text { Root } \\
\text { bark, } \\
\text { flowers, } \\
\text { leaf, } \\
\text { latex, } \\
\text { seeds } \\
\end{array}$ \\
\hline 10 & Asana & Pterocarpus marsupium Roxb. & $\begin{array}{c}\text { Tikta, } \\
\text { Kashay } \\
a\end{array}$ & $\begin{array}{l}\text { Laghu, } \\
\text { Rooksha }\end{array}$ & Sheeta & Katu & $\begin{array}{l}\text { Kapha, } \\
\text { pitta }\end{array}$ & $\begin{array}{l}\text { Heart- } \\
\text { wood, } \\
\text { exudate } \\
\text { resin }\end{array}$ \\
\hline 11 & Ashoka & Saraca asoca (Roxb.) DeWilde. & $\begin{array}{c}\text { Tikta, } \\
\text { Kashay } \\
a\end{array}$ & $\begin{array}{l}\text { Laghu, } \\
\text { Rooksha }\end{array}$ & Sheeta & Katu & $\begin{array}{l}\text { Kapha, } \\
\text { pitta }\end{array}$ & $\begin{array}{l}\text { Stem } \\
\text { bark, } \\
\text { seeds }\end{array}$ \\
\hline 12 & Aswatha & Ficus religiosa Linn. & $\begin{array}{c}\text { Madhur } \\
a, \\
\text { Kashay } \\
a\end{array}$ & $\begin{array}{c}\text { Guru } \\
\text { (Heavy } \\
\text { for diges- } \\
\text { tion), } \\
\text { Ruksha }\end{array}$ & Sheeta & Katu & $\begin{array}{l}\text { Kapha, } \\
\text { pitta }\end{array}$ & $\begin{array}{c}\text { Bark, } \\
\text { Tender } \\
\text { leaf, } \\
\text { Latex } \\
\text { and } \\
\text { Fruit }\end{array}$ \\
\hline 13 & Ativisha & $\begin{array}{l}\text { Aconitum heterophyllum Wall. ex } \\
\text { Royle. }\end{array}$ & $\begin{array}{l}\text { Katu, } \\
\text { Tikta } \\
\end{array}$ & $\begin{array}{l}\text { Laghu, } \\
\text { Rooksha }\end{array}$ & Ushna & Katu & $\begin{array}{l}\text { Kapha, } \\
\text { pitta }\end{array}$ & $\begin{array}{c}\text { Tuber- } \\
\text { ous root }\end{array}$ \\
\hline 14 & Badara & Ziziphus jujuba Mill. & $\begin{array}{l}\text { Madhur } \\
\quad a\end{array}$ & Guru & Sheeta & Katu & $\begin{array}{l}\text { Vata, } \\
\text { pitta }\end{array}$ & $\begin{array}{l}\text { Root, } \\
\text { leaf, } \\
\text { Fruit } \\
\end{array}$ \\
\hline 15 & Bhallataka & Semecarpus anacardium Linn. $f$. & $\begin{array}{c}\text { Katu, } \\
\text { Tikta, } \\
\text { Kashay } \\
a\end{array}$ & $\begin{array}{c}\text { laghu, } \\
\text { Teeksh- } \\
\text { na, } \\
\text { Snigdha }\end{array}$ & Ushna & $\begin{array}{l}\text { Madhu } \\
\text { ra }\end{array}$ & $\begin{array}{l}\text { Kapha, } \\
\text { Vata }\end{array}$ & Fruits \\
\hline 16 & Bharngi & $\begin{array}{l}\text { Clerodendrum serratum (Linn.) } \\
\text { Moon. }\end{array}$ & $\begin{array}{l}\text { Katu, } \\
\text { Tikta } \\
\end{array}$ & $\begin{array}{l}\text { Laghu, } \\
\text { Rooksha }\end{array}$ & Ushna & Katu & $\begin{array}{l}\text { Kapha, } \\
\text { Vata }\end{array}$ & $\begin{array}{l}\text { Root, } \\
\text { leaves }\end{array}$ \\
\hline 17 & Bhurja & Betula utilis D. Don. & $\begin{array}{c}\text { Kashay } \\
a \\
\end{array}$ & Laghu & Ushna & Katu & Tridosha & Bark \\
\hline 18 & Bhustrna & Andropogon citratus DC. & $\begin{array}{l}\text { Katu, } \\
\text { Tikta }\end{array}$ & $\begin{array}{l}\text { laghu, } \\
\text { Teekshna }\end{array}$ & Ushna & Katu & $\begin{array}{l}\text { Kapha, } \\
\text { Vata }\end{array}$ & $\begin{array}{l}\text { Leaf, } \\
\text { rhizome }\end{array}$ \\
\hline 19 & Bilwa & $\begin{array}{c}\text { Aegle marmelos (L.) Correa. ex } \\
\text { Roxb. }\end{array}$ & $\begin{array}{c}\text { Katu, } \\
\text { Tikta, } \\
\text { Kashay } \\
a \\
\end{array}$ & Teekshna & Ushna & Katu & $\begin{array}{l}\text { Kapha, } \\
\text { Vata }\end{array}$ & Fruits \\
\hline 20 & Bimbi & Coccinia indica $W$. and $A$. & Tikta & $\begin{array}{l}\text { Laghu, } \\
\text { Rooksha, } \\
\text { Teekshna }\end{array}$ & Ushna & Katu & $\begin{array}{l}\text { Kapha, } \\
\text { pitta }\end{array}$ & $\begin{array}{c}\text { Leaves, } \\
\text { Root, } \\
\text { Fruit }\end{array}$ \\
\hline 21 & Bruhati & Solanum indicum Linn. & $\begin{array}{r}\text { Katu, } \\
\text { Tikta }\end{array}$ & $\begin{array}{l}\text { Laghu, } \\
\text { Rooksha }\end{array}$ & Ushna & Katu & $\begin{array}{c}\text { Kapha, } \\
\text { Vata }\end{array}$ & $\begin{array}{c}\text { Root, } \\
\text { fruit }\end{array}$ \\
\hline
\end{tabular}


International Journal of Ayurvedic Medicine, 2017, 8(4), 159-168

\begin{tabular}{|c|c|c|c|c|c|c|c|c|}
\hline 22 & Chandana & Santalum album Linn. & $\begin{array}{l}\text { Tikta, } \\
\text { Madhura }\end{array}$ & $\begin{array}{l}\text { Laghu, } \\
\text { Rooksha }\end{array}$ & Sheeta & Katu & $\begin{array}{l}\text { Kapha, } \\
\text { pitta }\end{array}$ & $\begin{array}{c}\text { Heart } \\
\text { wood, } \\
\text { oil } \\
\end{array}$ \\
\hline 23 & Chavya & Piper chaba Hunter non.Blume. & Katu & $\begin{array}{l}\text { Laghu, } \\
\text { Rooksha }\end{array}$ & Ushna & Katu & $\begin{array}{c}\text { Kapha, } \\
\text { Vata }\end{array}$ & Roots \\
\hline 24 & Chirabilva & Holoptelia integrifolia Planch & $\begin{array}{l}\text { Tikta, } \\
\text { Kashaya }\end{array}$ & $\begin{array}{l}\text { Laghu, } \\
\text { Rooksha }\end{array}$ & Ushna & Katu & $\begin{array}{l}\text { Kapha, } \\
\text { pitta }\end{array}$ & Bark \\
\hline 25 & Chitraka & Plumbago zeylanica Linn. & Katu & $\begin{array}{l}\text { Laghu, } \\
\text { Rooksha }\end{array}$ & Ushna & Katu & $\begin{array}{c}\text { Kapha, } \\
\text { Vata }\end{array}$ & $\begin{array}{l}\text { Root } \\
\text { Bark }\end{array}$ \\
\hline 26 & Choraka & Angelica glauca Edgew. & $\begin{array}{l}\text { Katu, } \\
\text { Tikta }\end{array}$ & $\begin{array}{l}\text { Laghu, } \\
\text { Rooksha } \\
\end{array}$ & Ushna & Katu & $\begin{array}{c}\text { Kapha, } \\
\text { Vata }\end{array}$ & Root \\
\hline 27 & Darbha & Desmostachya bipinnata Stapf. & $\begin{array}{l}\text { Madhura, } \\
\text { Kashaya }\end{array}$ & $\begin{array}{l}\text { Laghu, } \\
\text { Snigdha }\end{array}$ & Sheeta & $\begin{array}{l}\text { Madhu } \\
\text { ra }\end{array}$ & Tridosha & Root \\
\hline 28 & $\begin{array}{l}\text { Da- } \\
\text { ruharidra }\end{array}$ & Berberis aristata $D C$. & $\begin{array}{l}\text { Tikta, } \\
\text { Kashaya }\end{array}$ & $\begin{array}{l}\text { Laghu, } \\
\text { Rooksha }\end{array}$ & Ushna & Katu & $\begin{array}{l}\text { Kapha, } \\
\text { pitta }\end{array}$ & $\begin{array}{l}\text { root, } \\
\text { stem, } \\
\text { fruit }\end{array}$ \\
\hline 29 & Devahva & Cedrus deodara (Roxb.) Loud. & $\begin{array}{c}\text { Katu, } \\
\text { Tikta, } \\
\text { Kashaya }\end{array}$ & $\begin{array}{l}\text { Laghu, } \\
\text { Rooksha }\end{array}$ & Ushna & Katu & $\begin{array}{l}\text { Kapha, } \\
\text { Vata }\end{array}$ & $\begin{array}{c}\text { Bark, } \\
\text { Heart- } \\
\text { wood, } \\
\text { oil }\end{array}$ \\
\hline 30 & Dhava & $\begin{array}{c}\text { Anogeissus latifolia Wall. ex } \\
\text { Bedd. }\end{array}$ & Kashaya & $\begin{array}{l}\text { Laghu, } \\
\text { Rooksha }\end{array}$ & sheeta & Katu & $\begin{array}{l}\text { Kapha, } \\
\text { pitta }\end{array}$ & $\begin{array}{c}\text { Heart } \\
\text { wood, } \\
\text { Exudate } \\
\text { and } \\
\text { Bark }\end{array}$ \\
\hline 31 & Ela & Elettaria cardamomum Maton. & $\begin{array}{l}\text { Madhura, } \\
\text { Katu, }\end{array}$ & $\begin{array}{l}\text { Laghu, } \\
\text { Rooksha }\end{array}$ & Sheeta & Katu & $\begin{array}{c}\text { Kapha, } \\
\text { Vata }\end{array}$ & Seeds \\
\hline 32 & Elavaluka & Prunus cerasus Linn. & $\begin{array}{l}\text { Tikta, } \\
\text { Kashaya }\end{array}$ & $\begin{array}{l}\text { Laghu, } \\
\text { Snigdha }\end{array}$ & Sheeta & Katu & $\begin{array}{l}\text { Kapha, } \\
\text { pitta }\end{array}$ & $\begin{array}{l}\text { Stem } \\
\text { bark, } \\
\text { Seeds } \\
\end{array}$ \\
\hline 33 & Hareetaki & Terminalia chebula Retz. & $\begin{array}{c}\text { Five } \\
\text { tastes } \\
\text { (except } \\
\text { lavana) }\end{array}$ & $\begin{array}{l}\text { Laghu, } \\
\text { Rooksha }\end{array}$ & Ushna & $\begin{array}{l}\text { Madhu } \\
\text { ra }\end{array}$ & Tridosha & Fruits \\
\hline 34 & Haridra & Curcuma longa Linn. & $\begin{array}{l}\text { Katu, } \\
\text { Tikta } \\
\end{array}$ & $\begin{array}{l}\text { Laghu, } \\
\text { Rooksha }\end{array}$ & Ushna & Katu & $\begin{array}{c}\text { Kapha, } \\
\text { Vata }\end{array}$ & $\begin{array}{l}\text { Rhi- } \\
\text { zome }\end{array}$ \\
\hline 35 & Hingu & Ferula foetida Regel. & Katu & $\begin{array}{l}\text { Laghu, } \\
\text { Snigdha } \\
\end{array}$ & Ushna & Katu & $\begin{array}{c}\text { Kapha, } \\
\text { Vata }\end{array}$ & Resin \\
\hline 36 & Ingudi & $\begin{array}{c}\text { Balanites aegyptiaca (Linn.) } \\
\text { Delile. }\end{array}$ & $\begin{array}{l}\text { Katu, } \\
\text { Tikta }\end{array}$ & $\begin{array}{l}\text { Laghu, } \\
\text { Snigdha }\end{array}$ & Ushna & Katu & $\begin{array}{l}\text { Kapha, } \\
\text { Vata }\end{array}$ & $\begin{array}{c}\text { Stem } \\
\text { bark, } \\
\text { fruit, } \\
\text { Seed oil }\end{array}$ \\
\hline 37 & Jambu & Syzygium cuminii (Linn.) Skeels. & $\begin{array}{l}\text { Madhura, } \\
\text { Amla, } \\
\text { Kashaya }\end{array}$ & $\begin{array}{l}\text { Laghu, } \\
\text { Rooksha }\end{array}$ & Sheeta & Katu & $\begin{array}{l}\text { Kapha, } \\
\text { pitta }\end{array}$ & $\begin{array}{l}\text { Fruit, } \\
\text { seed, } \\
\text { leaf, } \\
\text { stem } \\
\text { bark }\end{array}$ \\
\hline 38 & Jeeraka & Cuminum cyminum Linn. & Katu & $\begin{array}{c}\text { Laghu, } \\
\text { Rooksha }\end{array}$ & Ushna & Katu & $\begin{array}{c}\text { Kapha, } \\
\text { Vata }\end{array}$ & Seeds \\
\hline 39 & Jhingini & Odina wodier Roxb. & $\begin{array}{c}\text { Madhura, } \\
\text { Katu, } \\
\text { Kashaya }\end{array}$ & $\begin{array}{l}\text { Laghu, } \\
\text { Rooksha }\end{array}$ & Ushna & Katu & $\begin{array}{c}\text { Kapha, } \\
\text { Vata }\end{array}$ & $\begin{array}{l}\text { Bark, } \\
\text { leaves }\end{array}$ \\
\hline 40 & $\begin{array}{c}\text { Jyotish- } \\
\text { mati }\end{array}$ & Celastrus paniculatus Willd. & $\begin{array}{l}\text { Katu, } \\
\text { Tikta } \\
\end{array}$ & $\begin{array}{c}\text { Teeksh- } \\
\text { na }\end{array}$ & Ushna & Katu & $\begin{array}{c}\text { Kapha, } \\
\text { Vata }\end{array}$ & Seed oil \\
\hline 41 & Kadali & Musa paradisiaca Linn. & $\begin{array}{c}\text { Madhura, } \\
\text { Kashaya }\end{array}$ & $\begin{array}{c}\text { Guru, } \\
\text { Snigdha }\end{array}$ & Sheeta & $\begin{array}{c}\text { Madhu } \\
\text { ra }\end{array}$ & $\begin{array}{l}\text { Vata, } \\
\text { pitta }\end{array}$ & $\begin{array}{l}\text { Fruit, } \\
\text { Stem, } \\
\text { Flower }\end{array}$ \\
\hline 42 & Kadamba & Anthocephalus cadamba Miq. & $\begin{array}{l}\text { Tikta, } \\
\text { Kashaya }\end{array}$ & Rooksha & Sheeta & Katu & Tridosha & $\begin{array}{l}\text { Bark, } \\
\text { Fruit, } \\
\text { leaves } \\
\end{array}$ \\
\hline
\end{tabular}


Murali Krishna C et.al., Appraisal on Ayurvedic Herbs in the Management of Sthaulya (Obesity)

\begin{tabular}{|c|c|c|c|c|c|c|c|c|}
\hline 43 & Kalinga & Seeds of H. antidysenterica. & $\begin{array}{l}\text { Tikta, } \\
\text { Kashaya }\end{array}$ & $\begin{array}{l}\text { Laghu, } \\
\text { Rooksha }\end{array}$ & Sheeta & Katu & $\begin{array}{l}\text { Kapha, } \\
\text { pitta }\end{array}$ & Seeds \\
\hline 44 & Kantakari & Solanum xanthocarpum S. and $W$. & $\begin{array}{l}\text { Katu, } \\
\text { Tikta }\end{array}$ & $\begin{array}{l}\text { Laghu, } \\
\text { Rooksha }\end{array}$ & Ushna & Katu & $\begin{array}{l}\text { Kapha, } \\
\text { Vata }\end{array}$ & $\begin{array}{l}\text { whole } \\
\text { plant, } \\
\text { root, } \\
\text { fruit } \\
\end{array}$ \\
\hline 45 & Kapeetana & $\begin{array}{c}\text { Thespesia populnea Soland. ex } \\
\text { Correa. }\end{array}$ & Kashaya & $\begin{array}{l}\text { Laghu, } \\
\text { snigdha }\end{array}$ & Sheeta & Katu & $\begin{array}{l}\text { Kapha, } \\
\text { pitta }\end{array}$ & $\begin{array}{l}\text { Stem } \\
\text { bark }\end{array}$ \\
\hline 46 & Karanja & Derris indica (Lamk.) Bennet. & $\begin{array}{c}\text { Katu, } \\
\text { Tikta, } \\
\text { Kashaya }\end{array}$ & $\begin{array}{l}\text { Laghu, } \\
\text { Teeksh- } \\
\quad \text { na }\end{array}$ & Ushna & Katu & $\begin{array}{l}\text { Kapha, } \\
\text { Vata }\end{array}$ & $\begin{array}{c}\text { fruit, } \\
\text { leaves, } \\
\text { bark, } \\
\text { seeds }\end{array}$ \\
\hline 47 & $\begin{array}{l}\text { Kasamar- } \\
\quad d a\end{array}$ & Cassia occidentalis Linn. & $\begin{array}{l}\text { Madhura, } \\
\text { Tikta }\end{array}$ & $\begin{array}{l}\text { Laghu, } \\
\text { Rooksha }\end{array}$ & Ushna & Katu & Tridosha & $\begin{array}{l}\text { Root, } \\
\text { seed, } \\
\text { Leaf }\end{array}$ \\
\hline 48 & Katphala & Myrica nagi Hook. f. non-Thunb. & $\begin{array}{c}\text { Katu, } \\
\text { Tikta, } \\
\text { Kashaya }\end{array}$ & $\begin{array}{l}\text { Laghu, } \\
\text { Teeksh- } \\
\quad \text { na }\end{array}$ & Ushna & Katu & $\begin{array}{l}\text { Kapha, } \\
\text { Vata }\end{array}$ & $\begin{array}{l}\text { Bark, } \\
\text { Fruit }\end{array}$ \\
\hline 49 & Katuki & $\begin{array}{c}\text { Picrorhiza kurroa Royle. ex } \\
\text { Benth. }\end{array}$ & Tikta & $\begin{array}{l}\text { Laghu, } \\
\text { Rooksha }\end{array}$ & Sheeta & Katu & $\begin{array}{c}\text { Kapha, } \\
\text { Vata }\end{array}$ & $\begin{array}{l}\text { Root, } \\
\text { roots }\end{array}$ \\
\hline 50 & Khadira & Acacia catechu (Linn.f.) Willd. & $\begin{array}{l}\text { Tikta, } \\
\text { Kashaya }\end{array}$ & $\begin{array}{l}\text { Laghu, } \\
\text { Rooksha }\end{array}$ & Sheeta & Katu & $\begin{array}{l}\text { Kapha, } \\
\text { pitta }\end{array}$ & $\begin{array}{c}\text { bark, } \\
\text { heart } \\
\text { wood, } \\
\text { flowers }\end{array}$ \\
\hline 51 & Koshamra & Schleichera oleosa (Lour.) Oken. & Amla & Guru & Ushna & Katu & $\begin{array}{l}\text { Kapha, } \\
\text { Vata }\end{array}$ & $\begin{array}{l}\text { bark, } \\
\text { seed, oil }\end{array}$ \\
\hline 52 & Kramuka & Areca catechu Linn. & $\begin{array}{l}\text { Madhura, } \\
\text { Kashaya }\end{array}$ & $\begin{array}{l}\text { Guru, } \\
\text { Rooksha }\end{array}$ & Sheeta & Katu & $\begin{array}{l}\text { Kapha, } \\
\text { pitta }\end{array}$ & Fruit \\
\hline 53 & $\begin{array}{l}\text { Kuchan- } \\
\text { dana }\end{array}$ & Pterocarpus santalinus Linn. $f$. & $\begin{array}{l}\text { Madhura, } \\
\text { Tikta }\end{array}$ & $\begin{array}{l}\text { Guru, } \\
\text { Rooksha }\end{array}$ & Sheeta & Katu & $\begin{array}{l}\text { Kapha, } \\
\text { pitta }\end{array}$ & $\begin{array}{l}\text { heart } \\
\text { wood }\end{array}$ \\
\hline 54 & Kushta & Sausurea lappa Decne. & $\begin{array}{c}\text { Madhura, } \\
\text { katu, Tik- } \\
\text { ta }\end{array}$ & $\begin{array}{l}\text { Laghu, } \\
\text { Rooksha }\end{array}$ & Ushna & Katu & $\begin{array}{l}\text { Kapha, } \\
\text { Vata }\end{array}$ & Roots \\
\hline 55 & Kutaja & $\begin{array}{l}\text { Holarrhena antidysenterica } \\
\text { (Linn.) Wall. }\end{array}$ & $\begin{array}{l}\text { Tikta, } \\
\text { Kashaya }\end{array}$ & $\begin{array}{l}\text { Laghu, } \\
\text { Rooksha }\end{array}$ & Sheeta & Katu & $\begin{array}{l}\text { Kapha, } \\
\text { pitta }\end{array}$ & $\begin{array}{l}\text { Bark, } \\
\text { fruit }\end{array}$ \\
\hline 56 & Kutannata & Oroxylum indicum Vent. & $\begin{array}{c}\text { Madhura, } \\
\text { Tikta, } \\
\text { Kashaya }\end{array}$ & $\begin{array}{l}\text { Laghu, } \\
\text { Rooksha }\end{array}$ & Ushna & Katu & $\begin{array}{l}\text { Kapha, } \\
\text { Vata }\end{array}$ & $\begin{array}{l}\text { root } \\
\text { bark, } \\
\text { fruit }\end{array}$ \\
\hline 57 & Lodhra & Symplocos racemosa Roxb. & $\begin{array}{l}\text { Tikta, } \\
\text { Kashaya }\end{array}$ & $\begin{array}{l}\text { Laghu, } \\
\text { Rooksha }\end{array}$ & Sheeta & Katu & $\begin{array}{l}\text { Kapha, } \\
\text { pitta }\end{array}$ & $\begin{array}{l}\text { bark, } \\
\text { flower }\end{array}$ \\
\hline 58 & Madana & Randia dumetorum Poir. & $\begin{array}{l}\text { Madhura, } \\
\text { Tikta }\end{array}$ & $\begin{array}{l}\text { Laghu, } \\
\text { Rooksha }\end{array}$ & Ushna & Katu & $\begin{array}{c}\text { Kapha, } \\
\text { Vata }\end{array}$ & $\begin{array}{l}\text { Fruit, } \\
\text { seeds }\end{array}$ \\
\hline 59 & Madhooka & Bassia longifolia Koen. & $\begin{array}{l}\text { Madhura, } \\
\text { Kashaya }\end{array}$ & $\begin{array}{l}\text { Guru, } \\
\text { snigdha }\end{array}$ & Sheeta & $\begin{array}{l}\text { Madhu } \\
\text { ra }\end{array}$ & $\begin{array}{l}\text { Vata, } \\
\text { pitta }\end{array}$ & $\begin{array}{c}\text { Flow- } \\
\text { ers, } \\
\text { Seeds } \\
\text { and Oil }\end{array}$ \\
\hline 60 & Madhuka & Glycyrrhiza glabra Linn. & Madhura & $\begin{array}{l}\text { Guru, } \\
\text { snigdha }\end{array}$ & Sheeta & $\begin{array}{l}\text { Madhu } \\
\quad \text { ra }\end{array}$ & $\begin{array}{l}\text { Vata, } \\
\text { pitta }\end{array}$ & Roots \\
\hline 61 & Maricha & Piper nigrum Linn. & Katu & $\begin{array}{l}\text { Laghu, } \\
\text { Teeksh- } \\
\text { na, } \\
\text { sookshm } \\
\text { a }\end{array}$ & Ushna & Katu & $\begin{array}{l}\text { Kapha, } \\
\text { Vata }\end{array}$ & seeds \\
\hline
\end{tabular}


International Journal of Ayurvedic Medicine, 2017, 8(4), 159-168

\begin{tabular}{|c|c|c|c|c|c|c|c|c|}
\hline 62 & Mayuraka & Achyranthes aspera Linn. & $\begin{array}{l}\text { Katu, } \\
\text { Tikta }\end{array}$ & $\begin{array}{l}\text { Laghu, } \\
\text { Rooksha }\end{array}$ & Ushna & Katu & $\begin{array}{l}\text { Kapha, } \\
\text { Vata }\end{array}$ & $\begin{array}{l}\text { whole } \\
\text { plant, } \\
\text { Kshara }\end{array}$ \\
\hline 63 & Moorva & $\begin{array}{c}\text { Maerua arenaria Hook. f. and } \\
\text { Thoms. }\end{array}$ & $\begin{array}{l}\text { Tikta, } \\
\text { Kashaya }\end{array}$ & $\begin{array}{l}\text { Guru, } \\
\text { Rooksha }\end{array}$ & Ushna & Katu & Tridosha & Root \\
\hline 64 & Musta & Cyperus rotundus Linn. & $\begin{array}{c}\text { Katu, } \\
\text { Tikta, } \\
\text { Kashaya }\end{array}$ & $\begin{array}{l}\text { Laghu, } \\
\text { Rooksha }\end{array}$ & Sheeta & Katu & $\begin{array}{l}\text { Kapha, } \\
\text { pitta }\end{array}$ & Tuber \\
\hline 65 & Nagadanti & Baliospermum montanum Muell. & Katu & $\begin{array}{c}\text { Guru, } \\
\text { Teeksh- } \\
\text { na }\end{array}$ & Ushna & Katu & $\begin{array}{l}\text { Kapha, } \\
\text { vata }\end{array}$ & $\begin{array}{c}\text { Root, } \\
\text { Leaves, } \\
\text { Seed } \\
\end{array}$ \\
\hline 66 & Naktamala & Pongamia glabra Bent. & $\begin{array}{c}\text { Katu, } \\
\text { Tikta, } \\
\text { Kashaya }\end{array}$ & $\begin{array}{l}\text { Laghu, } \\
\text { Teeksh- } \\
\quad \text { na }\end{array}$ & Ushna & Katu & $\begin{array}{l}\text { Kapha, } \\
\text { Vata }\end{array}$ & $\begin{array}{c}\text { fruit, } \\
\text { leaves, } \\
\text { bark, } \\
\text { seeds }\end{array}$ \\
\hline 67 & Nyagrodha & Ficus benghalensis Linn. & Kashaya & $\begin{array}{l}\text { Guru, } \\
\text { Rooksha }\end{array}$ & Sheeta & Katu & $\begin{array}{l}\text { Kapha, } \\
\text { pitta }\end{array}$ & $\begin{array}{c}\text { Bark, } \\
\text { Tender } \\
\text { leaf, } \\
\text { Latex } \\
\end{array}$ \\
\hline 68 & Palasha & $\begin{array}{c}\text { Butea monosperma (Lam.) } \\
\text { Taub. }\end{array}$ & $\begin{array}{c}\text { Katu, } \\
\text { Tikta, } \\
\text { Kashaya }\end{array}$ & $\begin{array}{l}\text { Laghu, } \\
\text { Rooksha }\end{array}$ & Ushna & Katu & $\begin{array}{l}\text { Kapha, } \\
\text { Vata }\end{array}$ & $\begin{array}{c}\text { Flowers, } \\
\text { fruit, } \\
\text { Kshara }\end{array}$ \\
\hline 69 & Patha & Cissampelos pareira Linn. & Tikta & $\begin{array}{c}\text { Laghu, } \\
\text { Teeksh- } \\
\text { na }\end{array}$ & Ushna & Katu & $\begin{array}{l}\text { Kapha, } \\
\text { Vata }\end{array}$ & $\begin{array}{l}\text { Root } \\
\text { and } \\
\text { stem }\end{array}$ \\
\hline 70 & Pippali & Piper longum Linn. & Katu & $\begin{array}{l}\text { Laghu, } \\
\text { Teeksh- } \\
\text { na }\end{array}$ & Ushna & Katu & $\begin{array}{l}\text { Kapha, } \\
\text { Vata }\end{array}$ & Fruit \\
\hline 71 & $\begin{array}{l}\text { Pippali } \\
\text { mula }\end{array}$ & Roots of Piper longum Linn. & Katu & $\begin{array}{c}\text { Laghu, } \\
\text { Teeksh- } \\
\text { na }\end{array}$ & Ushna & Katu & $\begin{array}{l}\text { Kapha, } \\
\text { Vata }\end{array}$ & root \\
\hline 72 & Plaksha & Ficus lacor Buch.-Ham. & Kashaya & $\begin{array}{c}\text { Guru, } \\
\text { Rooksha }\end{array}$ & Sheeta & Katu & $\begin{array}{c}\text { Kapha, } \\
\text { pitta }\end{array}$ & Bark \\
\hline 73 & Prisniparni & Uraria picta Desv. & $\begin{array}{c}\text { Madhura, } \\
\text { Tikta }\end{array}$ & $\begin{array}{c}\text { Laghu, } \\
\text { Snigdha }\end{array}$ & Ushna & $\begin{array}{c}\text { Madhu } \\
\text { ra }\end{array}$ & Tridosha & Root \\
\hline 74 & Priyala & Buchanania lanzan Spreng. & Madhura & $\begin{array}{l}\text { Guru } \\
\text { sara } \\
\end{array}$ & Sheeta & $\begin{array}{c}\text { Madhu } \\
\text { ra }\end{array}$ & $\begin{array}{c}\text { Kapha, } \\
\text { pitta }\end{array}$ & $\begin{array}{l}\text { bark, } \\
\text { seed }\end{array}$ \\
\hline 75 & $\begin{array}{l}\text { Putikaran- } \\
\qquad j a\end{array}$ & Caesalpinia bonduc (L.) Roxb. & $\begin{array}{l}\text { Tikta, } \\
\text { Kashaya }\end{array}$ & $\begin{array}{l}\text { Laghu, } \\
\text { Rooksha }\end{array}$ & Ushna & Katu & Tridosha & $\begin{array}{l}\text { Root } \\
\text { bark, } \\
\text { leaf, } \\
\text { seed }\end{array}$ \\
\hline 76 & Rasna & Pluchia lanceolata Oliver. & Tikta & Guru & Ushna & Katu & $\begin{array}{l}\text { Kapha, } \\
\text { Vata }\end{array}$ & $\begin{array}{l}\text { Leaf, } \\
\text { Rhi- } \\
\text { zome }\end{array}$ \\
\hline 77 & Saireyaka & Barleria prionitis L. & $\begin{array}{c}\text { Madhura, } \\
\text { Tikta }\end{array}$ & Laghu & Ushna & Katu & $\begin{array}{c}\text { Kapha, } \\
\text { Vata }\end{array}$ & $\begin{array}{l}\text { Whole } \\
\text { plant }\end{array}$ \\
\hline 78 & Sarala & Pinus roxburghii Sarg. & $\begin{array}{c}\text { Katu, } \\
\text { Tikta, } \\
\text { Kashaya }\end{array}$ & $\begin{array}{c}\text { Laghu, } \\
\text { Snigdha, } \\
\text { Teeksh- } \\
\text { na }\end{array}$ & Ushna & Katu & $\begin{array}{l}\text { Kapha, } \\
\text { Vata }\end{array}$ & $\begin{array}{l}\text { Oil, } \\
\text { Bark, } \\
\text { resin, }\end{array}$ \\
\hline 79 & Sarja & Vateria indica Linn. & $\begin{array}{l}\text { Tikta, } \\
\text { Kashaya }\end{array}$ & Snigdha & Sheeta & Katu & $\begin{array}{l}\text { Kapha, } \\
\text { Vata }\end{array}$ & $\begin{array}{l}\text { Bark, } \\
\text { Gum }\end{array}$ \\
\hline 80 & Shaaka & Tectona grandis Linn. $f$. & $\begin{array}{l}\text { Tikta, } \\
\text { Kashaya }\end{array}$ & $\begin{array}{l}\text { Laghu, } \\
\text { Rooksha }\end{array}$ & Sheeta & Katu & $\begin{array}{l}\text { Kapha, } \\
\text { Vata }\end{array}$ & $\begin{array}{c}\text { Leaves, } \\
\text { seeds, } \\
\text { bark }\end{array}$ \\
\hline 81 & Shaala & Shorea robusta Gaertn. $f$. & $\begin{array}{l}\text { Madura, } \\
\text { Kashaya }\end{array}$ & Rooksha & Sheeta & Katu & $\begin{array}{l}\text { Vata, } \\
\text { pitta }\end{array}$ & $\begin{array}{l}\text { Bark, } \\
\text { heart- } \\
\text { wood }\end{array}$ \\
\hline 82 & Shalasara & Niryasa of Shaala & $\begin{array}{l}\text { Madura, } \\
\text { Kashaya }\end{array}$ & Rooksha & Sheeta & Katu & $\begin{array}{l}\text { Vata, } \\
\text { pitta }\end{array}$ & Resin \\
\hline
\end{tabular}




\begin{tabular}{|c|c|c|c|c|c|c|c|c|}
\hline 83 & Shatavari & Asparagus racemosa willd. & $\begin{array}{l}\text { Madhura, } \\
\text { Tikta }\end{array}$ & $\begin{array}{l}\text { Guru, } \\
\text { Snigdha }\end{array}$ & sheeta & $\begin{array}{l}\text { Madhu } \\
\quad r a\end{array}$ & $\begin{array}{l}\text { Vata, } \\
\text { pitta }\end{array}$ & $\begin{array}{c}\text { Tuber- } \\
\text { ous } \\
\text { roots }\end{array}$ \\
\hline 84 & Shigru & Moringa oleifera Lam. & $\begin{array}{l}\text { Katu, } \\
\text { Tikta }\end{array}$ & $\begin{array}{c}\text { Laghu, } \\
\text { Rooksha } \\
\text { ', } \\
\text { Teeksh- } \\
\text { na }\end{array}$ & Ushna & Katu & $\begin{array}{l}\text { Kapha, } \\
\text { Vata }\end{array}$ & $\begin{array}{c}\text { bark, } \\
\text { leaves, } \\
\text { fruits } \\
\text { and } \\
\text { seeds }\end{array}$ \\
\hline 85 & Shimshipa & Dalbergia sissoo Roxb. ex DC. & $\begin{array}{c}\text { Katu, } \\
\text { Tikta, } \\
\text { Kashaya }\end{array}$ & $\begin{array}{l}\text { Laghu, } \\
\text { Rooksha }\end{array}$ & Ushna & Katu & $\begin{array}{l}\text { Kapha, } \\
\text { Vata }\end{array}$ & $\begin{array}{c}\text { leaves, } \\
\text { Sara, } \\
\text { bark, } \\
\text { root }\end{array}$ \\
\hline 86 & Shireesha & Albizia lebbeck (Linn.) Willd. & $\begin{array}{c}\text { Madhura, } \\
\text { Tikta, } \\
\text { Kashaya }\end{array}$ & $\begin{array}{l}\text { Laghu, } \\
\text { Rooksha } \\
\text { Teeksh- } \\
\text { na }\end{array}$ & Ushna & Katu & $\begin{array}{l}\text { Tro- } \\
\text { dosha }\end{array}$ & $\begin{array}{c}\text { Bark, } \\
\text { seeds, } \\
\text { leaves } \\
\text { and } \\
\text { flowers }\end{array}$ \\
\hline 87 & Shunti & Zingiber officinalis Rosc. & Katu & $\begin{array}{c}\text { Guru, } \\
\text { Rooksha } \\
\text { ' } \\
\text { Teeksh- } \\
\text { na }\end{array}$ & Ushna & Katu & $\begin{array}{l}\text { Kapha, } \\
\text { Vata }\end{array}$ & $\begin{array}{l}\text { Rhi- } \\
\text { zome }\end{array}$ \\
\hline 88 & Siddharta & Brassica campestris Linn. & $\begin{array}{l}\text { Katu, } \\
\text { Tikta }\end{array}$ & $\begin{array}{c}\text { Laghu, } \\
\text { Snigdha }\end{array}$ & Ushna & Katu & $\begin{array}{c}\text { Kapha, } \\
\text { Vata }\end{array}$ & $\begin{array}{c}\text { Seed, } \\
\text { seed oil }\end{array}$ \\
\hline 89 & Surasa & Ocimum sanctum Linn. & $\begin{array}{l}\text { Katu, } \\
\text { Tikta }\end{array}$ & $\begin{array}{l}\text { Laghu, } \\
\text { Rooksha }\end{array}$ & Ushna & Katu & $\begin{array}{c}\text { Kapha, } \\
\text { Vata }\end{array}$ & $\begin{array}{l}\text { Leaf, } \\
\text { Root, } \\
\text { Seed }\end{array}$ \\
\hline 90 & Taala & Borassus flabellifer Linn. & Madhura & $\begin{array}{c}\text { Guru, } \\
\text { Snigdha }\end{array}$ & Sheeta & Katu & $\begin{array}{l}\text { Vata, } \\
\text { pitta }\end{array}$ & $\begin{array}{c}\text { Fruit, } \\
\text { Flower, } \\
\text { Juice, } \\
\text { Root }\end{array}$ \\
\hline 91 & Tarkari & Clerodendrum phlomidis Linn.f. & $\begin{array}{l}\text { Katu, } \\
\text { Tikta }\end{array}$ & $\begin{array}{l}\text { Laghu, } \\
\text { Rooksha }\end{array}$ & Ushna & Katu & $\begin{array}{l}\text { Kapha, } \\
\text { Vata }\end{array}$ & $\begin{array}{l}\text { Root } \\
\text { bark, } \\
\text { leaf }\end{array}$ \\
\hline 92 & Tinduka & Diospyros ebenum Koenig. & $\begin{array}{c}\text { Katu, } \\
\text { Tikta, } \\
\text { Kashaya }\end{array}$ & Guru & Sheeta & Katu & $\begin{array}{l}\text { Kapha, } \\
\text { pitta }\end{array}$ & $\begin{array}{l}\text { Bark, } \\
\text { fruit, } \\
\text { seed }\end{array}$ \\
\hline 93 & Tinisha & Ougeinia dalbergioides Benth. & Kashaya & $\begin{array}{l}\text { Laghu, } \\
\text { Rooksha }\end{array}$ & Sheeta & Katu & $\begin{array}{l}\text { Kapha, } \\
\text { pitta }\end{array}$ & $\begin{array}{c}\text { heart } \\
\text { wood } \\
\text { and } \\
\text { bark }\end{array}$ \\
\hline 94 & Udumbara & Ficus racemosa Linn. & Kashaya & $\begin{array}{c}\text { Guru, } \\
\text { Rooksha }\end{array}$ & Sheeta & Katu & $\begin{array}{l}\text { Kapha, } \\
\text { pitta }\end{array}$ & $\begin{array}{l}\text { Bark, } \\
\text { Fruit, } \\
\text { Latex }\end{array}$ \\
\hline 95 & Vacha & Acorus calamus Linn. & $\begin{array}{l}\text { Katu, } \\
\text { Tikta }\end{array}$ & $\begin{array}{c}\text { Laghu, } \\
\text { Teeksh- } \\
\text { na }\end{array}$ & Ushna & Katu & $\begin{array}{l}\text { Kapha, } \\
\text { Vata }\end{array}$ & $\begin{array}{l}\text { Rhi- } \\
\text { zome }\end{array}$ \\
\hline 96 & $\begin{array}{c}\text { Va- } \\
\text { jravruksha }\end{array}$ & $\begin{array}{c}\text { Euphorbia neriifolia auct. Non } \\
\text { Linn. }\end{array}$ & Katu & $\begin{array}{l}\text { Laghu, } \\
\text { Teeksh- } \\
\quad \text { na }\end{array}$ & Ushna & Katu & $\begin{array}{l}\text { Kapha, } \\
\text { Vata }\end{array}$ & $\begin{array}{l}\text { Root, } \\
\text { Leaf, } \\
\text { Milky } \\
\text { latex } \\
\end{array}$ \\
\hline 97 & Vanjula & Salix caprea Linn. & $\begin{array}{l}\text { Tikta, } \\
\text { Kashaya }\end{array}$ & Laghu & Sheeta & Katu & $\begin{array}{c}\text { Kapha,pi } \\
\text { tta }\end{array}$ & Flowers \\
\hline 98 & Varuna & Crataeva nurvala Buch.-Ham. & $\begin{array}{l}\text { Tikta, } \\
\text { Kashaya }\end{array}$ & $\begin{array}{l}\text { Laghu, } \\
\text { Rooksha }\end{array}$ & Ushna & Katu & $\begin{array}{l}\text { Kapha, } \\
\text { Vata }\end{array}$ & $\begin{array}{c}\text { Bark, } \\
\text { flower, } \\
\text { leaves }\end{array}$ \\
\hline 99 & Vibheetaki & Terminalia bellirica Roxb. & Kashaya & $\begin{array}{l}\text { Laghu, } \\
\text { Rooksha }\end{array}$ & Ushna & $\begin{array}{c}\text { Madhu } \\
\text { ra }\end{array}$ & $\begin{array}{c}\text { Kapha, } \\
\text { pitta }\end{array}$ & Fruits \\
\hline 100 & Vidanga & Embelia ribes Burm. $f$. & $\begin{array}{c}\text { Katu, } \\
\text { Kashaya }\end{array}$ & $\begin{array}{l}\text { Laghu, } \\
\text { Rooksha }\end{array}$ & Ushna & Katu & $\begin{array}{c}\text { Kapha, } \\
\text { Vata }\end{array}$ & $\begin{array}{c}\text { Fruits, } \\
\text { root }\end{array}$ \\
\hline
\end{tabular}


Table 3: List of anti-obesity herbs

\begin{tabular}{|c|c|c|c|}
\hline S.No. & Sanskrit name & Latin name & Family \\
\hline 1 & Agnimantha & Premna integrifolia Linn. & Verbenaceae \\
\hline 2 & Ajamoda & Trachyspermum roxburghianum (DC.) Craib. & Apiaceae \\
\hline 3 & Alarka & Calotropis gigantea (Linn.) R.Br. ex.Ait. & Apocynaceae \\
\hline 4 & Amalaki & Emblica officinalis Gaertn. & Phyllanthaceae \\
\hline 5 & Amra & Mangifera indica Linn. & Anacardiaceae \\
\hline 6 & Arjuna & Terminalia arjuna (Roxb.) W.\& $A$. & Combretaceae \\
\hline 7 & Arka & Calotropis procera (Ait.) R.Br. & Apocynaceae \\
\hline 8 & Asana & Pterocarpus marsupium Roxb. & Fabaceae \\
\hline 9 & Asoka & Saraca asoca (Roxb.) DeWilde. & Saraca asoca \\
\hline 10 & Aswatha & Ficus religiosa Linn. & Moraceae \\
\hline 11 & Badara & Ziziphus jujuba Mill. & Rhamnaceae \\
\hline 12 & Bhallataka & Semecarpus anacardium Linn. $f$. & Anacardiaceae \\
\hline 13 & Bilwa & Aegle marmelos (L.) Correa. ex Roxb. & Rutaceae \\
\hline 14 & Bimbi & Coccinia indica $W$. and $A$. & Cucurbitaceae \\
\hline 15 & Bruhati & Solanum indicum Linn. & Solanaceae \\
\hline 16 & Chandana & Santalum album Linn. & Santalaceae \\
\hline 17 & Chirabilva & Holoptelea integrifolia (Roxb.) Planch & Ulmaceae \\
\hline 18 & Chitraka & Plumbago zeylanica Linn. & Plumbaginaceae \\
\hline 19 & Darbha & Desmostachya bipinnata Stapf. & Poaceae \\
\hline 20 & Daruharidra & Berberis aristata DC. & Berberidaceae \\
\hline 21 & Devahva & Cedrus deodara (Roxb.) Loud. & Pinaceae \\
\hline 22 & Dhava & Anogeissus latifolia Wall. ex Bedd. & Combretaceae \\
\hline 23 & Hareetaki & Terminalia chebula Retz. & Combretaceae \\
\hline 24 & Haridra & Curcuma longa Linn. & Zingiberaceae \\
\hline 25 & Hingu & Ferula foetida Regel. & Umbelliferae \\
\hline 26 & Jambu & Syzygium cuminii (Linn.) Skeels. & Myrtaceae \\
\hline 27 & Jeeraka & Cuminum cyminum Linn. & Umbelliferae \\
\hline 28 & Kadali & Musa paradisiaca Linn. & Musaceae \\
\hline 29 & Kadamba & Anthocephalus cadamba Miq. & Rubiaceae \\
\hline 30 & Kalinga & Seeds of holarrhena antidysenterica L. & Apocynaceae \\
\hline 31 & Kantakari & Solanum xanthocarpum $S$. and $W$. & Solanaceae \\
\hline 32 & Kapeetana & Thespesia populnea Soland. ex Correa. & Malvaceae \\
\hline 33 & Karanja & Derris indica (Lamk.) Bennet. & Papilionoideae \\
\hline 34 & Kasamarda & Cassia occidentalis Linn. & Fabaceae \\
\hline 35 & Katuki & Picrorhiza kurroa Royle. ex Benth. & Plantaginaceae \\
\hline 36 & Khadira & Acacia catechu (Linn.f.) Willd. & Mimosaceae \\
\hline 37 & Kramuka & Areca catechu Linn. & Arecaceae \\
\hline 38 & Kuchandana & Pterocarpus santalinus Linn. $f$. & Fabaceae \\
\hline 39 & Kutannata & Oroxylum indicum Vent. & Bignoniaceae \\
\hline 40 & Lodhra & Symplocos racemosa Roxb. & Symplocaceae \\
\hline 41 & Madana & Randia dumetorum Poir. & Rubiaceae \\
\hline 42 & Madhooka & Bassia longifolia Koen. & Sapotaceae \\
\hline 43 & Madhuka & Glycyrrhiza glabra Linn. & Fabaceae \\
\hline 44 & Maricha & Piper nigrum Linn. & Piperaceae \\
\hline 45 & Mayuraka & Achyranthes aspera Linn. & Amaranthaceae \\
\hline 46 & Moorva & Maerua arenaria Hook. f. and Thoms. & Capparaceae \\
\hline 47 & Musta & Cyperus rotundus Linn. & Cyperaceae \\
\hline 48 & Naktamala & Pongamia glabra Bent. & Fabaceae \\
\hline 49 & Nyagrodha & Ficus benghalensis Linn. & Moraceae \\
\hline 50 & Palasha & Butea monosperma (Lam.) Taub. & Fabaceace \\
\hline 51 & Pippali & Fruits of Piper longum Linn. & Piperaceae \\
\hline
\end{tabular}




\begin{tabular}{|l|l|l|l|}
\hline 52 & Pippalimula & Roots of Piper longum Linn. & Piperaceae \\
\hline 53 & Putikaranja & Caesalpinia bonduc (L.) Roxb. & Fabaceace \\
\hline 54 & Shaaka & Tectona grandis Linn.. . & Lamiaceae \\
\hline 55 & Shatavari & Asparagus racemosa willd. & Asparagaceae \\
\hline 56 & Shigru & Moringa oleifera Lam. & Moringaceae \\
\hline 57 & Shimshipa & Dalbergia sissoo Roxb. ex DC. & Fabaceace \\
\hline 58 & Shireesha & Albizia lebbeck (Linn.) Willd. & Fabaceace \\
\hline 59 & Shunti & Zingiber officinalis Rosc. & Zingiberaceae \\
\hline 60 & Siddharta & Brassica campestris Linn. & Brassicaceae \\
\hline 61 & Surasa & Ocimum sanctum Linn. & Lamiaceae \\
\hline 62 & Tinduka & Diospyros ebenum Koenig. & Ebenaceae \\
\hline 63 & Tinisha & Ougeinia dalbergioides Benth. & Fabaceace \\
\hline 64 & Udumbara & Ficus racemosa Linn. & Moraceae \\
\hline 65 & Vacha & Acorus calamus Linn. & Acoraceae \\
\hline 66 & Varuna & Crataeva nurvala Buch. Ham. & Capparaceae \\
\hline 67 & Vibheetaki & Terminalia bellirica Roxb. & Combretaceae \\
\hline 68 & Vidanga & Embelia ribes Burm. f. & Primulaceae \\
\hline
\end{tabular}

\section{Discussion:}

Agni (digestive fire, etc) in the body has its primary contribution in metabolism. Ama (undigested toxic substance) which results from hypo functioning of Jatharagni (digestive fire) may obstruct the Srotas (channels) leading to Srotorodha (obstruction of channels). This leads to Medodushti along with decreases in the nutrient supply to subsequent Dhatus namely Asthi (bone tissue), Majja (bone marrow), and Shukra (fertility promoting substance). (22)

Charaka has given single Gana of 10 drugs (Lekhaniya Gana) Acharya Sushruta has given 8 Ganas, whereas Vagbhata included 10 Ganas to be Medohara. Surasadi Gana is not indicated for Medoroga by Sushruta while Vagbhata has included it. Laghu, Ruksha, Ushna, and Teekshna contribute for Langhana and Rukshaniya effect and plays predominant role for the treatment of vitiated Kapha Dosha and Medodhatu. According to Susruta the Vipaka of Langhana and Rukshaniya drug should be Laghu which is also interpreted as Katu Vipaka.

The Dravya possessing Katu Rasa and Tikta Rasa are to be prescribed in the initial stages (Border line of hyperlipidemia) in the treatment of Dyslipidemia. Later Kashaya dominant drugs can be incorporated in the subsequent phases (High hyperlipidemia) which facilitates for Shoshana (absorption) of liquefied or detoxified Kapha and Medas, a state produced by Tikta Rasa and Katu Rasa. Finally the application of Amla Rasa which is attributed with Vatanulomana (facilitator of downward movement of Vata), and Hridya (cardio-tonic) (23) properties may be preferred which may overcome Vataprakopa induced by Tikta, Katu, and Kashaya Rasa drugs.

Majority of herbs possessing Kaphahara, Vatahara and ushna veerya activity are found to be Medohara in action. Antagonistic measures are usually employed to treat Doshavriddhi (24). But in case of Medodushti, Sheeta Veerya dominant herbs are also suggested. Shalasaradi Gana, Lodhradi, and
Nyagrodhadi Ganas containing Kashaya, Tikta, and Sheeta Veerya drugs increase Ruksha Guna (dry) resulting in Medo soshana (absorption of vitiated fat). Drugs like Yashtimadhu (Glycyrrhiza glabra Linn.), Shatavari (Asparagus racemosus willd.), etc., possessing Madhura Rasa and Snigdha Guna are included in ganas. We can justify by saying they may help to soften and unction (25) the vessels hardened overtime by the deposited fat as in the case in Atherosclerosis.

It is observed that drugs like Guggulu (Commiphora wightii (Arn.) Bhandari.), Vrukshamla (Garcinia indica Choisy, Garcinia cambogia Desr.), Atasi (Linum usitassimum Linn.), Lashuna (Allium sativum Linn.) etc., promoted for controlling Obesity and Dyslipidemia though mentioned in Ayurvedic texts are not specifically found in classical Ganas.

\section{Conclusion:}

Drugs mentioned in each Gana of Ayurvedic classics have multiple pharmacological actions. This observation is useful for designing new formulations to treat Medodushti and its complications. Drugs that are Katu, Tikta, Kashaya in Rasa, possessing Ushna Virya, and Laghu Ruksha Guna are largely responsible for Medohara and Lekhaneeya activities in the treatment of Sthaulya. A better understanding in the existing evidence based science on herbs will further guide a qualitative research in obesity management that will attract the end users by the effective benefits. True randomized, double blinded, placebo-controlled clinical trials using herbal products will demonstrate their potential benefits.

\section{References:}

1. World Health Organization, "Obesity. Preventing and Managing the Global Epidemic, Report of a WHO Consultation (WHO Technical Report Series 894)," WHO, 2000. http://www.who.int/nutrition/ publications/obesity/WHO_TRS_894/en/ 
2. "Obesity and Overweight," Fact Sheet No. 311, 2012. http://www.who.int/mediacentre/factsheets/ fs311/en/index.html

3. Agnivesha, Charaka, Dridhabala, Charaka Samhita, Sutra Sthana, Ashtauninditeeya Adhyaya, 21/1, Vaidya Jadavaji Trikamji Aacharya. editor. 5th ed. Chaukhamba Sanskrit Sansthan, Varanasi; 2009; 116.

4. Agnivesha, Charaka, Dridhabala, Charaka Samhita, Sutra Sthana, Ashtauninditeeya Adhyaya, 21/3, Vaidya Jadavaji Trikamji Aacharya. editor. 5th ed. Chaukhamba Sanskrit Sansthan, Varanasi; 2009; 117.

5. Chakrapanidutta, Commentator, Sushruta Samhita, Sutra Sthana, Doshadhatumalakshayavruddhi Vijnaniya Adhyaya, 15/4, editor. Vaidya Jadavji Trikamji Acharya, 8th ed. Varanasi: Choukhambha Orientalia; 2005. p. 68.

6. Agnivesha, Charaka, Dridhabala, Charaka samhita, Sutra Sthana, Deerghanjeeviteeya Adhyaya, 1/61, Vaidya Jadavaji Trikamji Aacharya, editor, 5th ed. Chaukhamba Sanskrit Sansthan, Varanasi; 2009; 17.

7. Sushruta, Sushruta Samhita, Sutra Sthana, Doshadhatumalakshayavruddhi Vijnaniya Adhyaya, 15/4, editor Vaidya Jadavji Trikamji Acharya, 8th ed. Choukhambha Orientalia, Varanasi, 2005; 67.

8. Agnivesha, Charaka, Dridhabala, Charaka samhita, Sutra Sthana, Ashtauninditeeya Adhyaya, 21/4, Vaidya Jadavaji Trikamji Aacharya, editor, 5th ed. Chaukhamba Sanskrit Sansthan, Varanasi; 2009; 116.

9. Agnivesha, Charaka, Dridhabala, Charaka samhita, Nidana Sthana, Pramehanidanam Adhyaya, 4/51, Vaidya Jadavaji Trikamji Aacharya, editor, 5th ed. Chaukhamba Sanskrit Sansthan, Varanasi; 2009; 215.

10. Agnivesha, Charaka, Dridhabala, Charaka samhita, Sutra Sthana, Ashtauninditeeya Adhyaya, 4/3, Vaidya Jadavaji Trikamji Aacharya, editor, 5th ed. Chaukhamba Sanskrit Sansthan, Varanasi; 2009; 32.

11. Sushruta, Sushruta Samhita, Sutra Sthana, Dravyasangrahaneeyam Adhyaya, 38, editor Vaidya Jadavji Trikamji Acharya, 8th. Choukhambha Orientalia, Varanasi, 2005; 164-8.
12. Vagbhata, Ashtanga Samgraha, Sutra Sthana, Vividhadravyaganasangraha Adhyaya, 16, translated by Srikantha Murthy, 9th ed. Choukhambha Orientalia, Varanasi; 2005; 310.

13. Vagbhata, Ashtanga Hrudaya, Sutra Sthana, Shodhanadiganasangraha Adhyaya, 15, editor. Anna Moreshwar Kunte, Krishnashastri Navare, Harishastri, 9th ed. Choukhambha Orientalia, Varanasi, 2005; 229.

14. Dhanvantari Nighantu, editor. Sharma PV. 4th ed. Varanasi: Choukhambha Orientalia; 2005.

15. Bhavamishra, Bhava Prakasha Nigantu, edited Chunekar K.C, Pandeya G.S. Varanasi: Choukhambha Bharati Academy; 2006.

16. Vaidya Bapalal, Nighantu Adarsha. Vol. 1 and 2. Varanasi: Choukhambha Bharati Academy; 2005.

17. Nishteswar K, Hemadri K, Dravyaguna Vijnana, 1st ed. Delhi: Choukhambha Sanskrit Prathishthan; 2010.

18. Lucas Shanth Kumar, Dravyaguna Vijnana. Vol. 2, 1st ed. Varanasi: Choukhambha Visvabharati; 2008.

19. Sukh Dev, A selection of prime Ayurvedic plant drugs Ancient-modern concordance. New Delhi: Anamaya Publishers; 2006. p. 91.

20. Khare CP. Indian Medicinal Plants. Springer; 2007.

21. Sabnis Mukund, Chemistry and pharmacology of Ayurvedic medicinal plants. Varanasi: Chaukhambha Amarabharati Prakashan; 2006.

22. Agnivesha, Charaka, Dridhabala, Charaka Samhita, Sutra Sthana, Ashtauninditeeya Adhyaya, 21/4, Vaidya Jadavaji Trikamji Aacharya, editor. 5th ed. Chaukhamba Sanskrit Sansthan, Varanasi; 2009; 116.

23. Agnivesha, Charaka, Dridhabala, Charaka Samhita, Sutra Sthana, Atreyabhadrakapya Adhyaya, 26/43, Vaidya Jadavaji Trikamji Aacharya, editor. 5th ed. Chaukhamba Sanskrit Sansthan, Varanasi; 2009; 144.

24. Agnivesha, Charaka, Dridhabala, Charaka Samhita, Sutra Sthana, Deerghanjeeviteeya Adhyaya, 1/62, Vaidya Jadavaji Trikamji Aacharya, editor, 5th ed. Chaukhamba Sanskrit Sansthan, Varanasi; 2009; 17.

25. Agnivesha, Charaka, Dridhabala, Charaka Samhita, Sutra Sthana, Atreyabhadrakapya Adhyaya, 26/42, Vaidya Jadavaji Trikamji Aacharya, editor. 5th ed. Chaukhamba Sanskrit Sansthan, Varanasi; 2009; 143. 